\title{
Diffusion of vehicle exhaust pollutants in typical street canyons
}

\author{
Naiyan Zhan*, Zheng Gao, Yufeng Deng
}

School of Municipal and Environmental Engineering Department, Jilin Jianzhu University, Changchun 130118, China

Corresponding Author Email: 15504476322@163.com

https://doi.org/10.18280/ijht.360308

Received: 19 February 2018

Accepted: 15 July 2018

\section{Keywords:}

computational fluid dynamics (CFD),

street canyon, vortex, pollutant

concentration

\begin{abstract}
In view of the pollution caused by the growing car ownership in China and the complex layout of urban traffic system, this paper uses computational fluid dynamics (CFD) to simulate the air speed distribution and concentration of vehicle exhaust pollutants in five typical street canyons, including rising type, descending type, concave type, convex type and horizontal type. The simulation reveals certain differences in vortex structure and air speed among the five canyons. Among them, the pollutants may diffuse into the upstream in descending and concave canyons, but only spread to the downstream in the other three canyons. The upstream buildings have a far greater impact on pollutant concentration than downstream buildings. At the heights of $1.6 \mathrm{~m}$ and $10 \mathrm{~m}$, the rank of street canyons is descending > concave > rising > horizontal > convex in descending order in terms of the pollutant concentration.
\end{abstract}

\section{INTRODUCTION}

With the sustained economic growth and improvement of living standards, recent years have witnessed an upsurge in road transport demand and car ownership in China. By the end of June, 2017, the number of automobiles in China had exceeded 200 million [1]. The expansion of car ownership has brought convenience to daily lives, but also created serious environmental problems like exhaust pollution $[2,3]$.

Street canyon is the most polluted areas in urban road system. Due to the obstruction by roadside buildings, the exhaust gas from passing vehicles cannot diffuse smoothly but accumulate in the street canyon. In this area, the health of drivers and passengers, especially their respiratory system, is severely undermined by the exhaust of automobiles. The exhaust pollution in street canyon has been extensively studied by scholars at home and abroad. For instance, Yang et al. [4] simulated the diffusion of exhaust pollutants in a $2 \mathrm{D}$ street canyon, revealing the huge difference between pollutant concentration in windward and leeward areas at different heights at the constant diffusion rate. Zhu et al. [5] analysed the effect of wind speed, wind direction and building spacing on pollutant distribution in 3D street canyons. He et al. [6] examined the impact of symmetry on the airflow field in street canyons. Yassin et al. [7] suggested that the building shape directly bore on the pollutant diffusion in street canyon.

To sum up, most of these studies target 2D street canyons or 3D street canyons with relative regular building layout, failing to consider the influence of complex road and building structures on the road microenvironment. To make up for the gap, this paper applies computational fluid dynamics (CFD) to explore the internal flow field and pollutant concentration field in the street canyon of several typical urban buildings (e.g. rising and descending types) and discusses the law of pollutant diffusion inside the street canyon. The research findings provide insights into traffic organization, urban planning and many other issues.

\section{PHYSICAL AND MATHEMATICAL MODELS}

\subsection{Physical model}

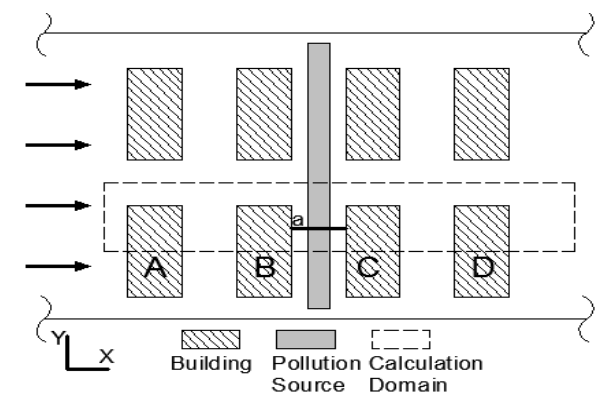

(a)

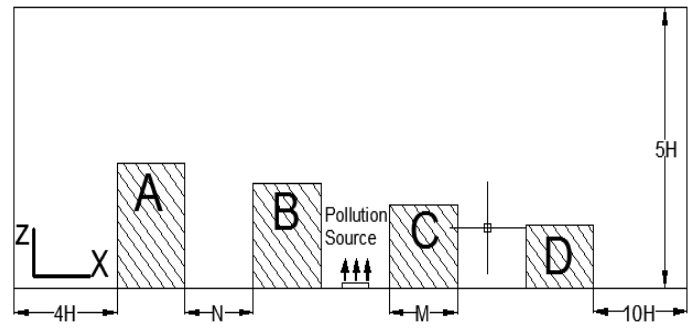

(b)

Figure 1. Top view and front view of the descending street canyon

A total of typical types of street canyons were simulated, namely, rising type, descending type, concave type, convex type and horizontal type. Figure 1 provides the top view and front view of the descending street canyon (Only use this figure for an example). In view of the symmetry of the model, the image method was only applied to the simulation of the phantom area, aiming to obtain accurate results within a short period of time [8]. In the calculation domain, the building length and width were set to $40 \mathrm{~m} \times 20 \mathrm{~m}(\mathrm{M})$, the maximum building height to $30 \mathrm{~m}(\mathrm{H})$, the street width to $20 \mathrm{~m}(\mathrm{~N})$, and 
the building spacing on the same side of the street canyon to $20 \mathrm{~m}$. Located in the middle of the street canyon, the pollutantemitting neighbourhood was assumed to be the pollution source (Along the direction of $\mathrm{Y}$ axis). The simulation parameters are listed in Table 1. If the calculation domain is too small, the end effect may cause errors in calculation results and distortion of simulation [9]. To solve this problem, the distances from the upstream building $\mathrm{A}$ and the downstream building $\mathrm{D}$ to the front of the calculation domain were set to $4 \mathrm{H}$ and $10 \mathrm{H}$, respectively; the height of the calculation domain was taken as $5 \mathrm{H}$. Through repeated simulation tests, the size of the calculation domain was determined as $560 \mathrm{~m} \times 150 \mathrm{~m} \times 50 \mathrm{~m}(\mathrm{X}, \mathrm{Y}, \mathrm{Z})$.

Table 1. Parameters in different types of street layout

\begin{tabular}{ccc}
\hline $\begin{array}{c}\text { Street Canyon } \\
\text { Form }\end{array}$ & $\begin{array}{c}\text { Building height: A D } \\
(\mathrm{m})\end{array}$ & $\begin{array}{c}\text { Building length } \\
(\mathrm{m})\end{array}$ \\
\hline $\begin{array}{c}\text { Horizontal type } \\
\text { Concave shaped } \\
\text { type }\end{array}$ & $20 ; 20 ; 20 ; 20$ & 40 \\
$\begin{array}{c}\text { Convex shaped } \\
\text { type }\end{array}$ & $25 ; 20 ; 20 ; 25$ & 40 \\
Rising type & $15 ; 20 ; 20 ; 15$ & 40 \\
Descending type & $15 ; 20 ; 25 ; 30$ & 40 \\
\hline
\end{tabular}

\subsection{Mathematical model}

Continuity equation:

$$
\frac{\overline{\partial \mathrm{u}_{\mathrm{i}}}}{\partial \mathrm{x}_{i}}=0
$$

Momentum equation:

$$
\frac{\partial \overline{\mathrm{u}_{\mathrm{i}}}}{\partial \mathrm{t}}+\overline{u_{j}} \frac{\partial \overline{u_{i}}}{\partial x_{j}}=-\frac{1}{\rho} \frac{\overline{\partial p}}{\partial x_{i}}+\frac{\partial}{\partial x_{j}}\left(v \frac{\overline{\partial u_{i}}}{\partial x_{j}}-\overline{u_{i}^{\prime} u_{j}^{\prime}}\right) \quad, \quad \mathrm{v}_{t}=c_{\mu} \frac{k^{2}}{\varepsilon}
$$

\section{$\mathrm{K}-\varepsilon$ equation:}

$$
\begin{aligned}
& \overline{u_{j}} \frac{\partial k}{\partial x_{j}}=\frac{\partial}{\partial x_{j}}\left(\frac{v_{t}}{\sigma_{k}} \frac{\partial k}{\partial x_{j}}+v \frac{\partial k}{\partial x_{j}}\right)+v_{t}\left(\frac{\overline{\partial u_{i}}}{\partial x_{j}}+\frac{\overline{\partial u_{j}}}{\partial x_{j}}\right) \frac{\overline{\partial u_{i}}}{\partial x_{j}}-\varepsilon \\
& \frac{\partial \varepsilon}{\partial \mathrm{t}}+\overline{\mathrm{u}_{\mathrm{j}}} \frac{\partial \varepsilon}{\partial \mathrm{x}_{j}}=\frac{\partial}{\partial x_{j}}\left(\frac{\partial \varepsilon}{\partial x_{j}} \frac{v_{t}}{\sigma_{\varepsilon}}\right)+\frac{\varepsilon}{k}\left[C_{\varepsilon 1} v_{t}\left(\frac{\overline{\partial u_{i}}}{\partial x_{j}}+\frac{\overline{\partial u_{j}}}{\partial x_{i}}\right) \frac{\overline{\partial u_{i}}}{\partial x_{j}}\right]-c_{\varepsilon 2} \frac{\varepsilon^{2}}{k}
\end{aligned}
$$

Pollutant convection diffusion equation:

$$
\frac{\partial\left(\mathrm{u}_{\mathrm{j}} c_{i}\right)}{\partial x_{j}}=\frac{\partial}{\partial x_{j}}\left(K \frac{\partial c_{i}}{\partial x_{j}}\right)+Q_{i}
$$

where $\rho$ is the air density; $\mathrm{t}$ is the time; $\mathrm{v}$ is the viscous coefficient of motion; $\mathrm{p}$ is the airflow pressure; $u^{i}$ and $u^{j}$ are the average speeds in different directions; $X_{i}$ is the component of Cartesian coordinates; $\mathrm{i}$ and $\mathrm{j}$ are in the range of $1 \sim 3$, with 1,2 and 3 being the $\mathrm{x}, \mathrm{y}$, and $\mathrm{z}$ direction components, respectively; $\overline{\mathrm{u}_{i}{ }^{\prime} u_{j}{ }^{\prime}}$ is the Reynolds stress tensor; $\delta_{i, j}$ is the Kronecker symbol; $v_{t}$ is the fluid force viscosity coefficient; $c_{\mu}=0.09, \mathrm{C}^{\varepsilon 1}=1.44, \mathrm{c}_{\varepsilon 2}=1.92, \sigma_{\mathrm{k}}=1.0$ and $\sigma_{\varepsilon}=1.0$, all of which are dimensionless $[10,11] ; \mathrm{k}$ is the turbulent kinetic energy; $\varepsilon$ is the turbulent dissipation rate; $\mathrm{C}^{\mathrm{i}}$ is the pollutant concentration at point i; $Q_{i}$ is the pollutant source.

\subsection{Boundary conditions}

In the calculation domain, the fluid includes the air and the polluting gas $\mathrm{CO}$, both of which are unsteady and incompressible. In light of this, the entrance of the calculation domain was set as a speed inlet (Along the arrow pointing direction in Figure 1. Besides, a user-defined function (UDF) was called from FLUENT to set the speed profile and the inlet wind speed as an exponential speed boundary condition $U(\mathrm{z})=U_{10^{\bullet}}(\mathrm{z} / 10)^{\curvearrowright}[12]$, with $U_{10}$ being the incoming wind speed $(3 \mathrm{~m} / \mathrm{s})$ at $10 \mathrm{~m}$ above the ground. The ground roughness coefficient a was set to 0.3 [13]. Considering its long distance from the block, the exit boundary was set as the outflow. The stationary ground and building wall of the calculation domain were simulated as non-slip boundaries (wall). The side and upper boundaries were simulated symmetrically with no convection flux or diffusion flux (symmetry) [14]. The pollution sources in the canyon were considered as continuous surface pollution sources located at the middle of the street canyon. The size of each source was determined as $8 \mathrm{~m}$-wide, $75 \mathrm{~m}$-long and $0.3 \mathrm{~m}$ above ground. $\mathrm{CO}$ is an important marker pollutant in city blocks, whose main source is the emission of urban vehicles. In the general near-ground environment, $\mathrm{CO}$ is relatively stable and it is not easy for $\mathrm{CO}$ to react chemically with other gases. Therefore, $\mathrm{CO}$ can be selected as a vehicle exhaust emission, and the emission speed of the main pollutant $\mathrm{CO}$ at the source $\mathrm{q}$ was set to $6 \times 10^{-5} \mathrm{~kg} / \mathrm{s}$ [15].

\subsection{Simulation methods}

The standard k- $\varepsilon$ turbulence model was selected considering its good overall prediction effect [16]. The model was established on Design Modeler and meshed by ANSYS. The grids were densified for the street canyon area and solved by FLUENT 16.0. The finite-volume method was employed to discretize the equations, following the second-order upwind plan. Moreover, the pressure and speed were coupled by the SIMPLE algorithm, and the equations were calculated iteratively by sub-relaxation method.

\section{ANALYSIS AND RESULTS DISCUSSION}

\subsection{Speed field distribution}

Figure 2 presents the distribution of airflow for different types of street canyons on the central section, i.e. along the ZX axis. Below are the detailed results on each type of street canyon. In the horizontal street canyon, three counterclockwise vortices of uniform size were observed in the canyons formed by Buildings $\mathrm{A}, \mathrm{B}, \mathrm{C}$ and $\mathrm{D}$, and the wall climbing effect was formed along the leeward buildings. The vortices were relatively obvious in the canyon between buildings $\mathrm{B}$ and $\mathrm{C}$ (the target canyon); in the concave street canyon, the airflow descended over Building $\mathrm{A}$ and the upper side of Building B. Large irregular vortices were formed on the upper side of upstream canyon (Buildings between $\mathrm{A}$ and B). Also, obvious vortices were observed in both the target canyon and downstream canyon (Buildings between $\mathrm{C}$ and $\mathrm{D}$ ), but the vertex centre in the deep blue low-speed area of the target canyon exhibited an obvious upward trend; in the 
convex street canyon, the airflow distribution was similar to that in the horizontal canyon. There was a clear vortex in the target canyon; in the rising street canyon, the airflow gradually rose along the building direction. Three vortices of different shapes were formed in the target canyon. The centre of each vortex was situated in the centre of the corresponding upwind building. The dark blue low-speed area was concentrated here; in the descending street canyon, the airflow distribution was significantly different from the other canyons: the airflow rose and passed over the large building A and then passed directly through the canyon. Finally, it flew over the top of the downstream building D. The descending street canyon was under the tail backflow zone formed by the airflow vortex on the top of Building A. A total of four vortices were formed with different shapes. The vortex of the target canyon appeared on the top of Building $\mathrm{C}$.

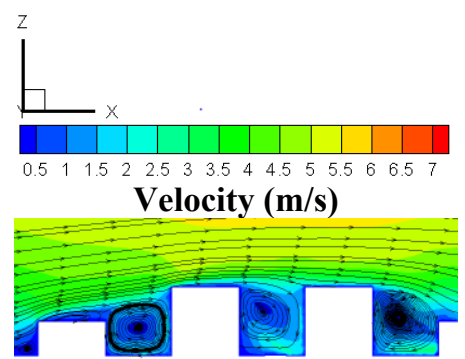

(c)Convex shaped street canyon

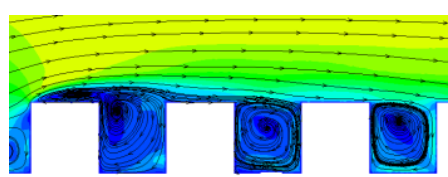

(a)Horizontal street canyon

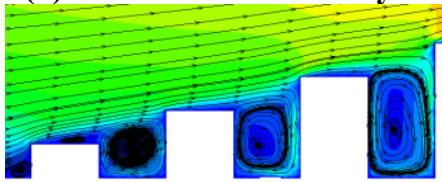

(d)Rising street canyon

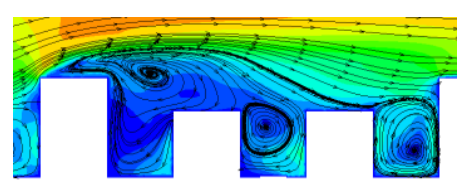

(b) Concave street canyon

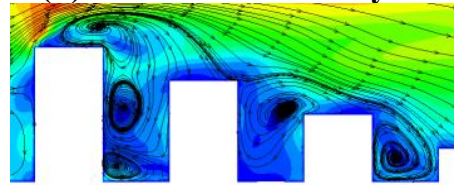

(e)Descending street canyon

Figure 2. Distribution of airflow for different types of street canyons on the central section

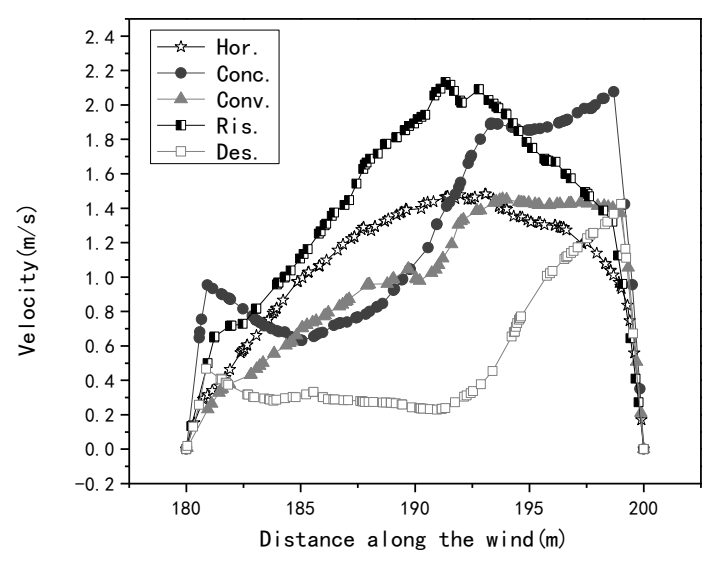

Figure 3. Windward variation of air speed at the height of $1.6 \mathrm{~m}$ along the central section of the target canyon

Then, the flow field distribution was investigated on the breathing plane (1.6m above ground) of the personnel in the target area, aiming to further compare the air speed variation of different types of street canyons. Figure 2 shows the windward variation of air speed at the height of $1.6 \mathrm{~m}$ along the central section of the target canyon (Line a in Figure 1). The results showed that the air speed of the target canyon generally increased first and then decreased; regular variation patterns could be discovered in the rising and horizontal street canyons, as verified by the relatively uniform vortices; in these two canyons, the vortex centre fell at the vertical centre of the street canyon; in other types of canyons, the vortex centre moved upwards and the airflow organization became more complicated on the target plane.

\subsection{Pollutant concentration field distribution}

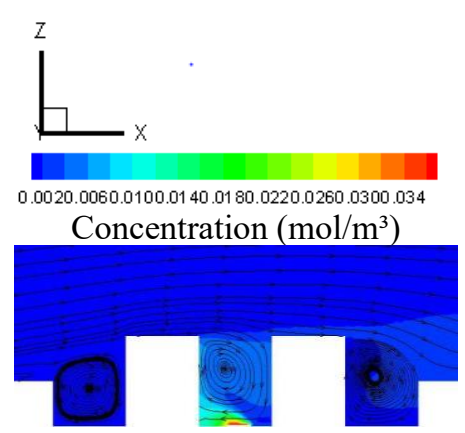

(c)Convex street canyon

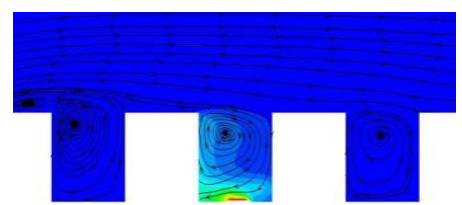

(a)Horizontal street canyon

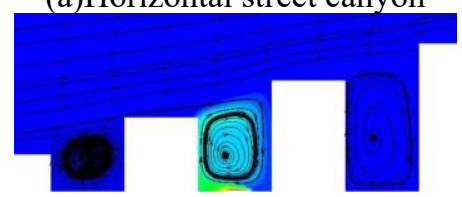

(d)Rising street canyon

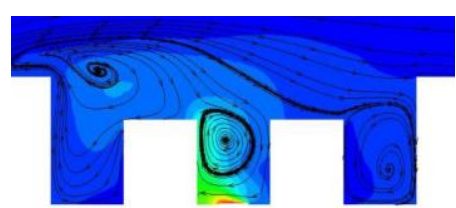

(b)Concave street canyon

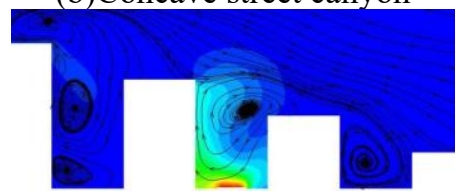

(e)Descending street canyon

Figure 4. Distribution of pollutant concentration under the street canyons on the central section

Figure 4 shows the distribution of pollutant concentration of the street canyons on the central section, i.e. along the $\mathrm{ZX}$ axis. As mentioned above, there are significant differences in the airflow distribution among the street canyons, owing to the variation in the height of roadside buildings. Hence, it is unsurprising that the street canyons differ greatly in the distribution of pollutant concentration. Below are the detailed results on each type of street canyon.

In the horizontal street canyon, the pollution diffusion was similar to that of the traditional street canyon with a height-to- width ratio of 1:1. The leeward pollutants were denser than windward pollutants, and the wall climbing effect was formed along the leeward building; in the concave street canyon, the vortex centre tended to move upwards. However, some pollutants were pushed back to the upstream by the upstream vortex, and some pollutants were carried by the airflow from the upstream to the downstream. Thus, there was a certain degree of pollutant accumulation in the downstream; in the convex street canyon, the pollutants in the target canyon concentrated on the leeward side and the canyon bottom. Some 
pollutants were diffused from the top side of the windward building to the downstream but did not accumulate on the leeward side in the downstream; in the rising street canyon, the pollutant distribution was similar to that of the convex canyon, except that the pollutants accumulated in the canyon under the effect of the tall buildings in the downstream. After all, it was difficult for the pollutants to diffuse out of the target canyon; in the descending street canyon, the situation was similar to that of the concave canyon. Under upstream reflux vortices, some pollutants were brought back to the upstream; under the rise of the vortex, the pollutants accumulated at the bottom of the target canyon.

In summary, the pollutants in the horizontal and convex street canyons were blown to the downstream by the dominant upstream wind. This movement was not seen in the rising street canyon. In addition to this movement, the pollutants in the concave and descending canyons were pushed back to the upstream by the reflux vortex at the top of upstream buildings. No pollutants accumulated in the rising street canyon due to the single speed streamline.

Next, the pollutant concentrations of each type of street canyon were compared at breathing height $(1.6 \mathrm{~m}$ above

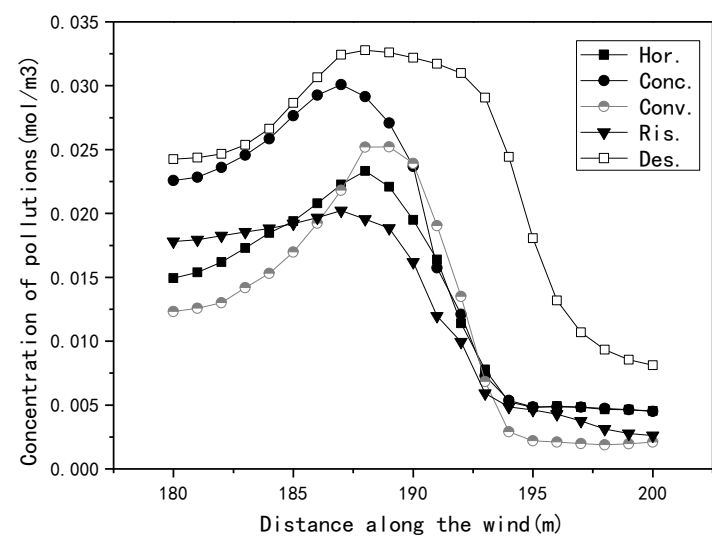

(a) 1.5 height ground) and $10 \mathrm{~m}$ height. Figure 5 showed the concentration distribution of pollutants at the heights of $1.6 \mathrm{~m}$ and $10 \mathrm{~m}$ in the wind direction along the central section of the target canyon; as shown in Figure 5 (a), the overall pollution concentration at the height of $1.6 \mathrm{~m}$ in the wind direction first increased and then decreased. This is because the section of $186 \mathrm{~m} \sim 194 \mathrm{~m}$ is a source of pollution emissions. Although the windward concentration is generally higher than leeward concentration, this section is so close to the source that it becomes a regional pollution zone; it can be seen from Figure 5 (b) that the highest concentration is discovered in concave and descending canyons, followed by rising canyon, horizontal canyon and convex canyon in descending order. In general, the pollutant concentration decreased monotonically along the wind direction at the height of $10 \mathrm{~m}$, and the pollutants were denser on the windward than on the leeward. The concentration at this height was basically the same as that at the height of $1.6 \mathrm{~m}$. The local deviations were resulted from the simulation error of the software. Therefore, At the heights of $1.6 \mathrm{~m}$ and $10 \mathrm{~m}$, the rank of street canyons was descending $>$ concave $>$ rising $>$ horizontal $>$ convex in descending order of the pollutant concentration.

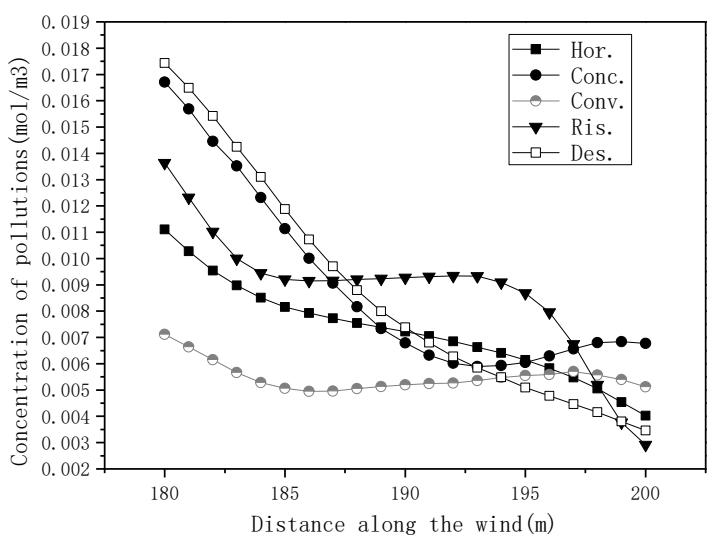

(b) $10 \mathrm{~m}$ height

Figure 5. Concentration distribution of pollutants in the wind direction along the central section of the target canyon

The above results demonstrate that the upstream buildings have a greater impact on pollutant distribution in target canyon than downstream buildings, and that the relative height of upstream buildings affects the relative air speed in the canyon. Therefore, the relative height of the upstream building is positively correlated with the pollutant concentration in the target canyon.

\section{CONCLUSIONS}

In view of the pollution caused by the growing car ownership in China and the complex layout of urban traffic system, this paper simulates the air speed distribution and concentration of vehicle exhaust pollutants in several typical street canyons. Through the simulation, the following conclusions can be drawn.

(1) The relative height of buildings determines the vortex structure and wind speed of different types of street canyons. Specifically, rising and horizontal canyons have relatively regular vortices, while the other three canyons differ greatly in vortex structure. As a result, these three canyons face great variation in air speed.

(2) In the target canyon, the pollutant flow is affected by the variation in upstream air streamline. The pollutants may diffuse into the upstream in descending and concave canyons, but only spread to the downstream in the other three canyons.

(3) The relative height of the upstream building affects the air speed in the canyon, which, in turn, alters the pollutant concentration at different heights. At the heights of $1.6 \mathrm{~m}$ and $10 \mathrm{~m}$, the rank of street canyons is descending $>$ concave $>$ rising $>$ horizontal $>$ convex in descending order of the pollutant concentration.

\section{REFERENCES}

[1] Liu ZY. (2017). The Law Network of China.

[2] Fan YV, Klemeš JJ, Lee CT. (2018). Pre- and posttreatment assessment for the anaerobic digestion of lignocellulosic waste: p-graph. Chemical Engineering Transactions 63:

$1-6$. https://doi.org/10.3303/CET1863001

[3] Iervolino G, Casson Moreno V, Tugnoli A, Cozzani V. (2018). Carbon balance of a waste biomass supply chain: the integration of a pyrolysis-based valorization process. Chemical Engineering Transactions 65: 1-6. https://doi.org/10.3303/CET1865001 
[4] Yang H, Zhang Q, Zhu H. (2017). Numerical simulation of the diffusion of vehicle pollutants in two dimensional urban street canyon. Journal of Guilin College of Areospace Technology 22(4): 375-381. https://doi.org/10.3969/j.issn.1009-1033.2017.04.005

[5] Zhu JH, Li DY, Li F. (2016). Study on airflow structures and poluutant distribution in urban treet canyons. Building Energy \& Environment 35(9): 47-51.

[6] He ZN, Gao YH. Li YH, Li ZS. (2008). Effects of symmetry of urban street canyons on their inner airflow fields. Journal of meteorology and environment 24(2): 62-67. https://doi.org/10.3969/j.issn.1673503X.2008.02.015

[7] Yassin MF. (2011). Impact of height and shape of building roof on air quality in urban street canyons. Atmospheric Environment 45(29): 5220-5229. https://doi.org/10.1016/j.atmosenv.2011.05.060

[8] Hang J, Li Y. (2010). Wind conditions in idealized building clusters e macroscopic simulations using a porous turbulence model. Boundary-Layer Meteorology 136(1): 129-159. https://doi.org/10.1007/s10546-0109490-3

[9] Wang FJ. (2004). Computational fluid mechanics analysis-Principles and applications of CFD software. Beijing: Tsinghua University press.
[10] Launder BE, Spalding DB. (1972) Lectures in mathematical models of turbulence. Academic Press 169.

[11] Sini JF, Anquetiin S, Mestayer PG. (1996). Pollutant dispersion and thermal effects in urban street canyons. Atmospheric Environment 30(15): 2659-2677. https://doi.org/10.1016/1352-2310(95)00321-5

[12] Sang JG, Wang BM, Tong H, Liu HZ. (2002). Numerical simulation of the flow field and thermal structure over a street canyon. Journal of Applied Meteorological Science (z1): 69-81. https://doi.org/10.3969/j.issn.10017313.2002.z1.008

[13] Barratt R. (2001). Atmospheric dispersion modelling. An introduction to practical applications. London: Earthscan Publication.

[14] Shi BZ. (1993). Environmental impact assessment of construction projects. China environmental science press.

[15] Wu Y, Hao JM, Fu LX. (2002). The list of motor vehicle emissions in Macao. Journal of Tsinghua University (Science and Technology) 42(12): 1601-1604.

[16] Huang YD, Wang SS, Jin X, Sun YN, Jin MX. (2008). A comparative study of various turbulence models for simulating pollutant dispersion within an urban street canyons. Chinese Journal of Hydrodynamics 23(2): 189195. 